\title{
ESTIMACIÓN DE UNA MATRIZ ORIGEN-DESTINO A PARTIR DE LA TELEFONÍA MÓVIL Y OTROS DATOS DE FUENTES HETEROGÉNEAS
}

\author{
Luis Miguel Romero Pérez \\ Universidad de Sevilla, España \\ Noelia Cáceres Sánchez \\ AICIA, España \\ Francisco García Benítez \\ Universidad de Sevilla, España \\ Antonio Reyes Gutiérrez \\ Universidad de Sevilla, España
}

\section{RESUMEN}

La información obtenida de forma directa de una encuesta domiciliaria, basada en el hogar, no puede considerarse, en general, fidedigna a nivel de pares O-D. Los viajes capturados se expanden para reproducir el número de viajes totales y los generados por las zonas de transporte. A través de ese proceso de expansión se concentran los viajes generados por las zonas de transporte en un pequeño conjunto de relaciones O-D capturadas en la encuesta. Por el contrario, las virtudes de una matriz O-D derivada de la telefonía móvil son varias: relativamente rápida de obtener y económicamente muy competitiva frente a una nueva encuestación, está basada en observaciones de una muestra amplia de la población y no tiene riesgo de sesgo ni de una baja tasa de respuesta. Sin embargo, por su naturaleza, tiene dificultades para captar los viajes de corta duración. Esta comunicación combina la información derivada de la telefonía móvil con la obtenida mediante encuestas domiciliarias, conteos de tráfico y datos de tiempos de viaje para obtener una matriz Origen-Destino representativa de hora punta de la Ciudad de Málaga y los municipios integrados en el ámbito territorial del Consorcio de Transporte Metropolitano del Área de Málaga (CTMAM), junto a Torremolinos y Benalmádena por estar enclavados en el ámbito territorial del consorcio. El trabajo ha sido desarrollado en el marco de un proyecto de investigación para la Agencia de Obra Pública de la Junta de Andalucía. La comunicación presenta aspectos novedosos en cuanto a la información de partida, matrices de telefonía móviles y datos de tiempo de viaje de Google para todos los arcos de la red, tanto como en la metodología desarrollada para la completa integración de dicha información de naturaleza heterogénea.

\section{INTRODUCCIÓN}

En los estudios de Movilidad hay una cantidad ingente de información a la que hay que conceder tan solo cierto grado de fiabilidad. Al enfrentar toda la información disponible se observan incongruencias entre algunos datos. Es necesario proceder a un detenido análisis 
de las posibles causas de tales incoherencias. Un claro ejemplo es la discrepancia observada entre los volúmenes de vehículos medidos en la realidad (puntos aforados en la red) y los modelados a través de la matriz Origen-Destino. La práctica habitual en los estudios de consultoría es atribuir exclusivamente dicha discrepancia a una incorrecta definición de la matriz O-D, por lo que se procede sin más a modificarla. Sin embargo, dicha matriz es obtenida por medio de costosos procesos de encuestación de la población y da una idea bastante aproximada de la magnitud de los viajes agregados sobre las zonas de transporte o ZT. Cualquier distorsión excesiva de dicha información invalida el esfuerzo presupuestario y humano dedicado a la recopilación de la información de movilidad. Esto es de especial relevancia en el proyecto DIURMOVIL (AOPJA, 2015), que ha dado origen a esta comunicación. En el citado proyecto se utilizan fuentes de información heterogéneas, cada una con sus características propias y todas de un valor indudable. En especial se dispone de dos matrices previas, la matriz obtenida de la encuesta domiciliaria 2010 homogeneizada, EDM2010 homogeneizada, y la obtenida a través de un operador de telefonía móvil. En base a este razonamiento, en la definición de una metodología de estimación de una matriz Origen-Destino más fiable se ha alcanzado un compromiso entre la distorsión de la solución con relación a la matrices previas y a los volúmenes observados a nivel agregado en determinados arcos de la red. El objetivo perseguido es diseñar un algoritmo tal que, partiendo de una matriz de demanda desactualizada y otra proveniente de un operador de telefonía móvil estime una Matriz de Viajes Ajustada que, cuando ésta sea asignada a la red, genere unos flujos modelados que se asemejen a los volúmenes medidos en los arcos.

En la práctica, actualizar una matriz referencia utilizando datos de volúmenes medidos en los aforos es un proceso muy económico. Se parte de una matriz ya existente y del conocimiento de un conjunto, más o menos extenso, de datos de aforos en las vías principales que las administraciones recogen automáticamente. Durante los últimos 40 años esta metodología ha sido la opción más atractiva y ha recibido especial atención en la literatura técnica dedicada a la planificación del transporte.

Si se denota por $\bar{A}$ el subconjunto de arcos aforados del conjunto $A$ de arcos de la red para el periodo objeto de estudio, la obtención de la matriz O-D para dicho periodo se plantea inicialmente en términos del sistema de ecuaciones (1):

$$
v_{a}=\sum_{i \in T Z} \sum_{j \in T Z} p_{i j, a} \cdot T_{i j}=\bar{v}_{a} \quad \forall a \in \bar{A} \subseteq A
$$

donde $\bar{v}_{a}$ denota el volumen de tráfico (aforo) soportado y medido por el arco genérico $a$, $v_{a}$ el volumen de tráfico modelado a través de la proporción de viajes $p_{i j, a}$ soportado por el arco $a$ proveniente de la pareja origen-destino $i$-j frente al total de viajes que lo atraviesa, y $T_{i j}$ el número de viajes asociado a la pareja $i-j$ de la matriz OD. Ordenando los elementos 
de la matriz de viajes $T_{i j}$ en forma de un pseudovector $t_{q}$, donde cada subíndice $q$ representa un par O-D perteneciente al conjunto $P$ de todos los pares O-D de la red, la expresión anterior se escribe como:

$$
v_{a}=\sum_{q \in P} p_{q, a} \cdot t_{q}=\bar{v}_{a} \quad \forall a \in \bar{A} \subseteq A
$$

El tratamiento de la matriz de viajes como pseudovector hace más simple y comprensible las expresiones. En apartados sucesivos se emplearán indistintamente las variables $T_{i j}$ y $t_{q}$ aunque se continúan nombrando como matriz de viajes. En general, como nomenclatura referente a una matriz de viajes se usará una letra mayúscula cuando la expresión se refiera a la forma matricial y minúscula en formato pseudovector. Para hacer mención de un elemento específico de la matriz, se usarán dos subíndices en el primer caso (primer subíndice para el origen de los viajes, segundo para el destino), y uno en el caso del elemento del pseudovector (subíndice del par O-D).

El sistema de ecuaciones planteado en (1) o (2) es generalmente incompatible. Los valores recogidos en los puntos de aforo presentan inconsistencias que llevan a un problema de ajuste sin solución. Estas inconsistencias se deben a errores en el proceso de medición como son: $i$ ) las mediciones en los puntos de aforo pueden realizarse en diferentes horas, incluso días, ii) el número de mediciones tomadas en cada aforo es diferente, teniendo más fiabilidad cuantas más horas de medición se encuentren disponibles, iii) existe tráfico recursivo imposible de discriminar, $i v$ ) el sistema de aforo puede arrojar valores erróneos, etc.

Para salvar los problemas de incompatibilidad e indeterminación, los métodos de ajuste se formulan como problemas de optimización matemática. Así, se selecciona la mejor solución, entre un conjunto de soluciones, maximizando o minimizando un criterio determinado. Puede concluirse que los distintos métodos para el problema de estimación de matrices de viajes a partir de volúmenes de tráfico desarrollados en la literatura, presentan la forma genérica siguiente (Yang et al, 1992):

$$
\begin{array}{cl}
\underset{\boldsymbol{t}, \boldsymbol{v}}{\operatorname{Minimizar}} & F(\boldsymbol{t}, \boldsymbol{v})=\gamma_{1} F_{1}(\boldsymbol{t}, \bar{t})+\gamma_{2} F_{2}(\boldsymbol{v}, \overline{\boldsymbol{v}}) \\
\text { s.a. } \quad \boldsymbol{v} & =M(\boldsymbol{t}) \boldsymbol{t} \\
& \boldsymbol{t} \in \Omega
\end{array}
$$

siendo $\gamma_{1}$ y $\gamma_{2}$ factores de peso, con $\gamma_{1} \geq 0, \gamma_{2} \geq 0, \gamma_{1}+\gamma_{2}=1$. Las restricciones (4) representan el modelo de asignación. Cada fila de la matriz $M(t)$ está formada por las fracciones de viajes correspondientes a cada par O-D para los diferentes arcos aforados. $\Omega$ es el conjunto de las matrices O-D factibles, normalmente definido por las restricciones de no negatividad de los elementos aunque es posible añadirle ciertas restricciones lineales como la limitación de las generaciones y atracciones de los centroides. 
Las funciones $F_{1}(\boldsymbol{t}, \overline{\boldsymbol{t}}) F_{2}(\boldsymbol{v}, \overline{\boldsymbol{v}})$ representan medidas de distancia generalizadas entre la matriz O - D estimada $\boldsymbol{t}$ y la matriz de partida $\overline{\boldsymbol{t}}$, y entre el flujo modelado en el arco $\boldsymbol{v}$ y el observado $\overline{\boldsymbol{v}}$. En resumen, se minimiza la discrepancia respecto de dos parámetros $\boldsymbol{t}$ y $\boldsymbol{v}$ con el fin de realizar el ajuste de la matriz de viajes. Se supone que las funciones son convexas, por lo general definidas como cuadráticas, entrópicas o de máxima probabilidad y están diseñadas para tener en cuenta la aleatoriedad de la información recogida.

Los factores de peso $\gamma_{1}$ y $\gamma_{2}$ en (3) reflejan la confianza (o incertidumbre) en la información contenida en $\overline{\boldsymbol{t}}$ y $\overline{\boldsymbol{v}}$. Si la matriz previa se considera más fiable y precisa en comparación con los conteos de tráfico, se establece $\gamma_{1} \gg \gamma_{2}$, y se obtiene como resultado una matriz muy cercana a la previa donde se acepta una mayor desviación entre los volúmenes modelados y los observados; en el caso contrario, $\gamma_{1} \ll<\gamma_{2}$, la matriz previa no tendrá influencia y los volúmenes estimados serán muy similares a los observados. Existe un caso especial cuando la matriz previa es fiable y los conteos de tráfico se consideran libres de errores. Entonces la función objetivo de la formulación (3)-(5) se reduce a $F(\boldsymbol{t}, v)=F_{1}(\boldsymbol{t}, \overline{\boldsymbol{t}})$

Se puede clasificar la estimación de matrices O-D en dos categorías según si las proporciones de viajes en arco dependen de la matriz de viajes o no. Existe un elevado número de referencias bibliográficas en cada categoría. En el caso de asignación proporcional las restricciones (4) se formulan como:

$$
v=[\Pi] \cdot t
$$

donde $\Pi$ es la matriz de proporciones, en este caso constante durante todo el proceso.

\section{PROPIEDADES DEL PROBLEMA DE ESTIMACIÓN DE MATRICES}

\subsection{Zonificación de la región de estudio}

Las matrices O-D contienen los viajes agregados de todos los usuarios que deciden de forma individual viajar desde una zona origen hacia otra zona destino. Desde otro punto de vista, las matrices O-D representan el número de viajeros que se originan y atraen entre las diversas zonas de la red de transportes del área en estudio: la Ciudad de Málaga y los municipios integrados en el ámbito territorial del Consorcio de Transporte Metropolitano del Área de Málaga (CTMAM), junto a Torremolinos y Benalmádena por estar enclavados en el ámbito territorial del consorcio.

Al realizar la zonificación de la red, se establece la hipótesis de que las características de cada zona se concentran en un único punto llamado centroide y se agregan sus características socioeconómicas. La división en zonas se realiza manteniendo el esqueleto principal de la red. Los arcos contenidos dentro de cada división son simplificados o sustituidos por conectores. Estos últimos son los que enlazan los centroides con la red, sus propiedades son 
diferentes a los demás arcos.

Una de las fuentes de información de este proyecto, la facilitada por el operador de telefonía móvil, parte de una zonificación basada en criterios diferentes a la zonificación del modelo de transporte. Su criterio para agrupar en zonas no es la homogeneidad de las variables propias de movilidad, sino la de mantenimiento de la calidad de sus servicios y cobertura. Para la generación de matrices Origen-Destino a través de datos de telefonía es necesario un proceso previo de compatibilización de zonas de telefonía y zonas de transporte, en el que establezca las relaciones necesarias para definir los movimientos de los usuarios de telefonía en función de ZT (zona de transporte) de Origen y de ZT de Destino. En cualquier caso, con el objeto de mantener la confidencialidad de la geometría y configuración de su red de antenas y celdas de telefonía móvil, la matriz proveniente del operador es suministrada en su formato final, con la conversión entre zonas de Telefonía a ZT ya llevada a cabo y sin posibilidad de refinamiento ni de ningún tipo de intervención en dicho proceso.

Otra posible fuente de incertidumbre se origina en relación a los desplazamientos intrazonales, aquellos que tienen su origen y destino en la misma ZT. En el proceso de asignación de la matriz OD al modelos de red, los viajes intrazonales no cargan la red, pues atendiendo a la definición del modelo de transporte, aquellos no llegan a salir del centroide de la zona, sin llegar a asignarse a ningún arco del modelo de red. Sin embargo, en la realidad estos desplazamientos usan el viario y pueden recogerse en los aforos utilizados por el ajuste. Para intentar adaptarse a una medición observada en un punto aforado con una componente intrazonal importante, el proceso de ajuste tenderá a incrementar los viajes de un par O-D que atraviese dicho arco, introduciendo por tanto una distorsión artificial en la matriz Origen-Destino ajustada.

\subsection{Periodo de tiempo para el que se define la matriz de viajes}

Desde el punto de vista de la oferta de la infraestructura viaria, el servicio facilitado por la red suele permanecer casi constante durante largos periodos de tiempo salvo que aparezcan problemas que disminuyan su capacidad como pueden ser accidentes, aparición de estacionamiento en doble fila, etc; en cambio la demanda de vehículos está en constante variación a lo largo del día. Los usuarios acceden a la red desde diferentes situaciones y en diferentes instantes de tiempo. En cada momento del día se refleja una matriz de viajes distinta sobre la red. Puede afirmarse por tanto que los sistemas de transporte en la práctica no están en estado de equilibrio de forma permanente, aunque generalmente se establece la hipótesis de que están cerca del equilibrio o tendiendo hacia dicho estado.

Al igual que las agregaciones de viajes por zonas, es necesario agregar dicha información por periodos de tiempo con el fin de simplificar y reducir la complejidad del problema. Según la longitud del periodo de tiempo considerado el proyecto puede afrontarse desde un punto de vista estático o dinámico. 
Si el periodo de tiempo considerado en el proyecto es amplio (como en este caso, hora punta de mañana), el problema se analiza desde un punto de vista estático. En este caso se suponen las mismas condiciones de demanda durante dicho periodo de tiempo y estarán representadas por una única matriz de viajes. El ajuste que se analiza en este proyecto es de la matriz de hora punta de mañana, establecida en el periodo de 8:00-8:59, correspondiente a la situación más desfavorable de máxima carga en la red de transporte.

\subsection{Indeterminación del problema}

En la región de estudio la zonificación y la red cohabitan en el mismo espacio-temporal, de tal manera que la accesibilidad entre cualquier pareja de zonas origen-destino está asegurada por la red modelada, de tal manera que cada individuo puede acceder desde su centroide origen hasta cualquier centroide destino diferente del de salida (excluyendo los viajes internos en cada zona). El número de combinaciones entre centroides, eliminando los viajes intra-zonales, es $N^{2}-N$, siendo $\mathrm{N}$ el número total de zonas de transporte definidas; de donde el número de rutas es al menos $N$-1 veces el número de zonas. Este valor indica el número de elementos de la matriz de viajes que conforman las variables del problema y que, generalmente, resultan varios órdenes superior al número de arcos de la red. Sabiendo que cada arco aforado aporta una restricción lineal al problema del ajuste, puede demostrarse que, aun aforando el conjunto íntegro de arcos, el sistema es indeterminado. La medición del volumen de vehículos en todos los arcos de la red es inviable, en la práctica se suele aforar aproximadamente un $10 \%$ del total. Además, el aforado de todos los arcos es innecesario, puede no aportar más información al problema debido a que existen dependencias lineales entre ellos. A través de las leyes de Kirchhoff o de conservación del flujo en los nodos, existen tantas relaciones entre arcos como nodos internos tenga el grafo.

A esta indeterminación, hay que añadirle un problema que aporta mayor complejidad a la estimación de matrices. El comportamiento de los usuarios es individualista ya que su objetivo a la hora de elegir ruta no es el de disminuir el tiempo total del sistema sino el suyo propio. Cada usuario selecciona las calles, carreteras o vías principales evitando las que le consuman un mayor tiempo de viaje. Esto hace que los usuarios de un gran número de pares O-D utilicen las mismas vías, las principales. Con este comportamiento no todos los arcos de la red son utilizados, acumulando un gran número de vehículos las vías principales haciendo complicada la discriminación de los porcentajes de viajeros de cada par O-D en los arcos.

Para ilustrar lo anterior se estudia la indeterminación de la red de Málaga. La red se compone de 6.153 arcos y 1.779 nodos, de los cuales 178 son centroides. La matriz de viajes por tanto consta de 31.506 elementos (existen del orden de 5 veces más pares que arcos en la red). Se tienen medidas de conteos de tráfico en 212 arcos, lo que supone un $3.5 \%$ respecto al total de arcos. Por tanto, en este problema concreto, el sistema de ecuaciones planteado en ¡Error! No se encuentra el origen de la referencia. o (2) tiene 212 ecuaciones para 31.506 incógnitas, lo que supone un sistema altamente indeterminado. 


\subsection{Proceso de asignación}

En el problema de asignación de tráfico de vehículos a una red de transporte, en adelante $T A P$, el coste de una ruta (e.g. tiempo si no hay peajes en la red, coste generalizado en otro caso) depende del volumen de vehículos que circulan por los arcos de la red. El coste de viaje en una ruta es la suma del coste de cada uno de los arcos que la componen, por tanto función del volumen de vehículos que circulan por cada arco. Los arcos son comunes a las diferentes rutas que unen los pares Origen-Destino de la región en estudio. Así, el flujo de una ruta carga todos los arcos que la componen, con lo que está influyendo en el coste del resto de rutas a través de los arcos que comparte con ellas. La relación coste / volumen o intensidad de tráfico que circula por el arco viene caracterizada por la función de demora, que puede ser modelada mediante una determinada forma funcional (e.g. BPR, cónicas) dependiendo de una serie de parámetros. Éstos pueden ser en general de dos tipos:

- Unos parámetros característicos de la forma funcional: en general un vector de parámetros $\boldsymbol{\alpha}$ que controlan la forma que responde el arco ante la congestión;

- y características funcionales del arco que determinan el nivel de intensidad de vehículos que se alcanza en la congestión: número de carriles, capacidad de cada carril, nivel de doble fila o de minoración de la capacidad, grado de tráfico de agitación, etcétera.

El patrón de flujo sobre la red de transporte responde a un equilibrio entre la congestión en los arcos y la decisión de viajar de los conductores por una determinada ruta. Como resultado final del problema de asignación se obtienen los flujos en los arcos que se encuentran en equilibrio con el tiempo o coste generalizado de atravesar dichos arcos. Analizando la estabilidad del problema, se define la solución final como un estado de equilibrio estable. Esto es, cuando el problema se encuentra en dicho estado no existen atracciones actuando sobre él que lo hagan evolucionar hacia otro estado diferente. Si el problema no está en equilibrio, se generan atracciones que hacen tender la solución hacia un estado en equilibrio estable. Estos factores, que actúan sobre el sistema, son los mecanismos de cambio de rutas de los usuarios, desde su ruta actual hasta otra más competitiva. En el equilibrio, no existirá ningún incentivo para cambiarse de ruta.

El problema más extendido y usado en la modelización de la movilidad en el ámbito de un área metropolitana es un caso particular de la asignación simétrica, en el que el coste del arco $s_{a}$ depende únicamente de su propio flujo:

$$
s_{a}(v)=s_{a}\left(v_{a}\right) \quad \forall a \in A
$$

Considerando el viaje, desde un origen a un destino, como una actividad intermedia necesaria para cumplir la actividad principal en destino, es razonable considerar que cada 
usuario tendrá un comportamiento egoísta tratando de minimizar su propio coste de viaje para poder desarrollar su actividad principal. Así, cada conductor actuará de forma independiente escogiendo la ruta que menor coste le suponga. Con este comportamiento, desde el punto de vista del colectivo de viajeros, se alcanzará un estado en el que ningún viajero puede mejorar su coste de viaje mediante el cambio de ruta. Éste es un estado en el que se cumple la condición de equilibrio de usuario, UE: user-equilibrium (Wardrop, 1952).

Si el problema de asignación de tráfico supone que, además de tener información completa acerca de las diferentes rutas posibles, los usuarios perciben el mismo coste de viaje bajo las mismas condiciones, se está resolviendo el problema de equilibrio de usuario determinista. Este problema es el más extendido en la asignación de tráfico y es uno de los más utilizados en el problema de ajuste de matrices origen-destino.

El principal inconveniente del equilibrio de usuario determinista es que supone una perfecta, o al menos uniforme, previsión (con cero errores de percepción) del coste de viaje por parte de los usuarios. En la realidad, estas percepciones en los costes están sujetas a variaciones. Una generalización del equilibrio de usuario determinista es el problema de equilibrio de usuario estocástico, SUE (Sheffi, 1985). En el equilibrio de usuario estocástico se relaja la hipótesis de información perfecta por lo que no se tiene una percepción idéntica de los costes. En este caso se supone que todos los usuarios conocen la red con igual incertidumbre;el coste percibido es considerado como una variable aleatoriamente distribuida entre los conductores. Así, en vez de tener una ruta con menor coste de viaje, existe un conjunto de rutas que pueden seleccionarse como de menor coste. La solución encontrada en el equilibrio de usuario estocástico representa una mejor descripción de la realidad observada. Además tiene la ventaja, respecto del equilibrio de usuario determinista, que tanto la solución en términos de flujos en arcos como de flujos en rutas es única.

La asignación determinista basada en el equilibrio del usuario es muy sensible a variaciones en el coste de los arcos. Cualquier variación en el coste de una ruta puede hacer que ésta deje de ser competitiva y por tanto deje de ser usada, descargando todos los arcos que la componen. Sin embargo, pese al papel fundamental que juegan las funciones de demora en este mecanismo de reparto de flujo entre rutas alternativas, a su definición y posterior calibrado se le suele asignar pocos recursos. En muchos casos puede observarse que las funciones de demora utilizadas en un estudio provienen de una familia de funciones diseñadas y definidas para otro con dudosa similitud. Se utilizan en su mayor parte los mismos valores numéricos para los parámetros, con lo que no se están considerando las particularidades de la nueva región metropolitana. También es una práctica muy habitual utilizar directamente las funciones de demora por defecto del software de asignación para gran parte de la red (por ejemplo, funciones BPR con parámetros $\alpha=0.15 \mathrm{y} \beta=4$ ). Esto desvirtúa el proceso de asignación, que es matemáticamente muy complejo, ya que en la definición de un vector de flujos en el conjunto de todos los $\operatorname{arcos} A$ intervienen una gran cantidad de parámetros y variables. Es posible, debido a ello, perder el sentido físico del 
proceso de asignación, que consiste simplemente en repartir el flujo entre pares O-D por las diferentes rutas que las unen atendiendo al tiempo de viaje de cada una de ellas. En este proyecto se ha propuesto una simplificación del proceso de asignación, distribuyendo el flujo entre las diferentes rutas no por medio del tiempo de viaje modelado a través de las funciones de demora y sus asociadas fuentes de incertidumbre, sino utilizando los tiempos de viaje observados, recopilados a través del Directions Service de la API de Google Maps. Con esta aproximación al problema se pierde, durante todo el proceso de ajuste de la matriz O-D, la dependencia entre la demanda de viajes y su coste, que se producía a través del proceso de equilibrado de tiempo de viajes entre las rutas usadas. Sin embargo, tras esta medida propuesta no subyace la hipótesis de considerar ambos fenómenos independientes, sino simplemente el hecho de considerar que los mecanismos que regulan la relación entre ambos no son conocidos, aunque sí su resultado. Usar los tiempos observados en lugar de los modelados aporta muchas ventajas, como la de considerar componentes de tráfico que tradicionalmente están deficientemente recogidas en los modelos de redes de transporte, como el tráfico intrazonal (i.e. misma ZT origen y destino del viaje), el tráfico de paso (i.e. vehículos cuyo origen y destino no pertenecen a la zonificación) y el tráfico de agitación (i.e. vehículos que dan vueltas en torno a su destino en búsqueda de aparcamiento), no incluidos en las matrices Origen-Destino pero sí medidos en los aforos. Como principal desventaja a priori, cabe señalar que se pierde la capacidad de reproducir los resultados del ajuste en un software comercial basado en los algoritmos clásicos deterministas. En este trabajo se ha abordado la determinación de la matriz O-D que mejor reproduce la realidad observada relajando la dependencia de los tiempos de las rutas con respecto a la matriz de demanda, posponiendo para un proceso futuro el cálculo de los parámetros de las funciones de demora que mejor representan el comportamiento observado. Operativamente, el principal atractivo de usar los tiempos de viaje observados en lugar de los obtenidos mediante las funciones de demora, con una asignación tipo estocástica con el algoritmo STOCH (Dial, 1971), que no requiere enumeración de caminos y por tanto es computacionalmente eficiente, es que al mantenerse constante los tiempos de viaje en los arcos a lo largo de todo el proceso puede considerarse un caso de proporciones constantes, tal como la formulada por (9)

Conviene destacar una vez más que lo que se mantiene es el modo en el que se reparten los viajes entre las diferentes rutas reales, teniendo como referencia el reparto real observado. El cálculo de la matriz de proporciones no es inmediato, sino que tiene que hacerse a través de sucesivas asignaciones a la red de transporte de un viaje para cada par origen-destino. Una vez calculada, la matriz de proporciones permanece constante hasta que se considere que los tiempos de viaje, observados a través del Directions Service de Google Maps con los que se ha calculado, han experimentado un cambio sustancial y ya no son representativos de la red para el periodo de estudio.

\subsection{Recogida de información en la estimación de matrices}

La fase de recopilación y captura de información puede llevarse a cabo a través de diversas 
acciones enfocadas a reducir el coste y tiempo de la estimación de la matriz de viajes. A continuación se exponen las más profusamente empleadas. Todas ellas pueden combinarse para obtener una mayor calidad de los datos de entrada. En los siguientes apartados se destacan las características de la información de partida que más influyen en el diseño del algoritmo de ajuste.

\subsubsection{Matriz de viajes base}

Si está disponible, es muy recomendable partir de una matriz O-D base o de partida, generada para el sistema de transportes en un proyecto anterior al que actualmente se acomete. La matriz O-D estará evidentemente desactualizada, pero puede contener información muy valiosa en cuanto a su estructura. Antes de utilizar una matriz desactualizada es necesario comprobar la fuente de procedencia, antigüedad y esfuerzo económico que supuso para poder así evaluar su fiabilidad o "confianza” respecto del resto de información disponible.

\subsubsection{Encuestas de viajes}

La realización de encuestas sobre una muestra estadísticamente representativa de la población es una técnica muy empleada. La información que se obtiene es de mucha calidad ya que puede conocerse la hora y motivo del viaje, ruta seleccionada, etc. para cada familia encuestada. La principal desventaja de este método es que el hecho de responder a una encuesta consume tiempo y puede constituir una intromisión a la privacidad, por lo que además del coste de recogida de la información, es necesario luego realizar una corrección y validación de los datos. Debido a ello, la encuestación domiciliaria en un área metropolitana se lleva a cabo con una periodicidad mayor en general a los 5 años, por lo que el riesgo de que su contenido esté desactualizado es elevado. Además, es importante resaltar un efecto poco mencionado, aunque muy conocido, como es la incertidumbre y errores que se generan debido al proceso de expansión de los valores de los individuos encuestados al universo de la población estudiada. La muestra se diseña habitualmente teniendo en cuenta la pirámide poblacional de las zonas de transporte para elegir un conjunto de individuos representativos de su zona. Así, y dado el presupuesto disponible para su ejecución, se define el tamaño muestral correspondiente a cada zona para alcanzar un nivel de significancia adecuado. Esa representatividad de las variables muestrales puede aplicarse tan solo a magnitudes zonales (el nivel de desagregación para el que se diseñó la muestra) o incluso variables referidas a toda la región de estudio, como pueden ser el número de viajes totales o los viajes generados por las zonas. Los coeficientes de elevación de los viajes han sido calculados considerando solo magnitudes de la zona origen, no del par O-D específico de los viajes descritos en la encuesta. Por ello, dada una región dividida en un conjunto $Z$ de ZTs, de dimensión $N$, los viajes capturados en la encuestación para cada uno de los $N^{2}$ parejas O-D no son significativos de la relación entre dicho par. No es económicamente viable entrevistar al porcentaje mínimo necesario de viajeros que realicen un viaje entre cada par O-D para obtener variables significativas de dicho flujo. Por tanto, la información obtenida de forma directa de una encuesta domiciliaria, basada en el hogar, no puede considerarse fidedigna a nivel de pares O-D. Los viajes capturados han sido expandidos para reproducir 
el número de viajes totales y los generados por las zonas de transporte. Esto genera que la estructura de la matriz expandida contenga muchos ceros (relaciones O-D no interceptadas durante el proceso de encuestación). Para una zona de la región en estudio, $i \in Z$, una muestra de usuarios de dicha zona, a la que se aplica los coeficientes de expansión correspondientes a cada uno de los usuarios de la muestra, da lugar a un total de viajes $O_{i}$ generados por dicha zona. La muestra de usuarios de la zona $i$ entrevistados ha recogido viajes de un subconjunto $\mathrm{P}$ de pares OD con origen del viaje en $i$. A través del proceso de expansión de la muestra se están concentrando el total de viajes $O_{i}$ generado por dicha zona en los pares capturados en la encuesta, cuando en realidad estaría distribuido entre todos los pares $i$-j, con $j \in Z / j \neq i$.

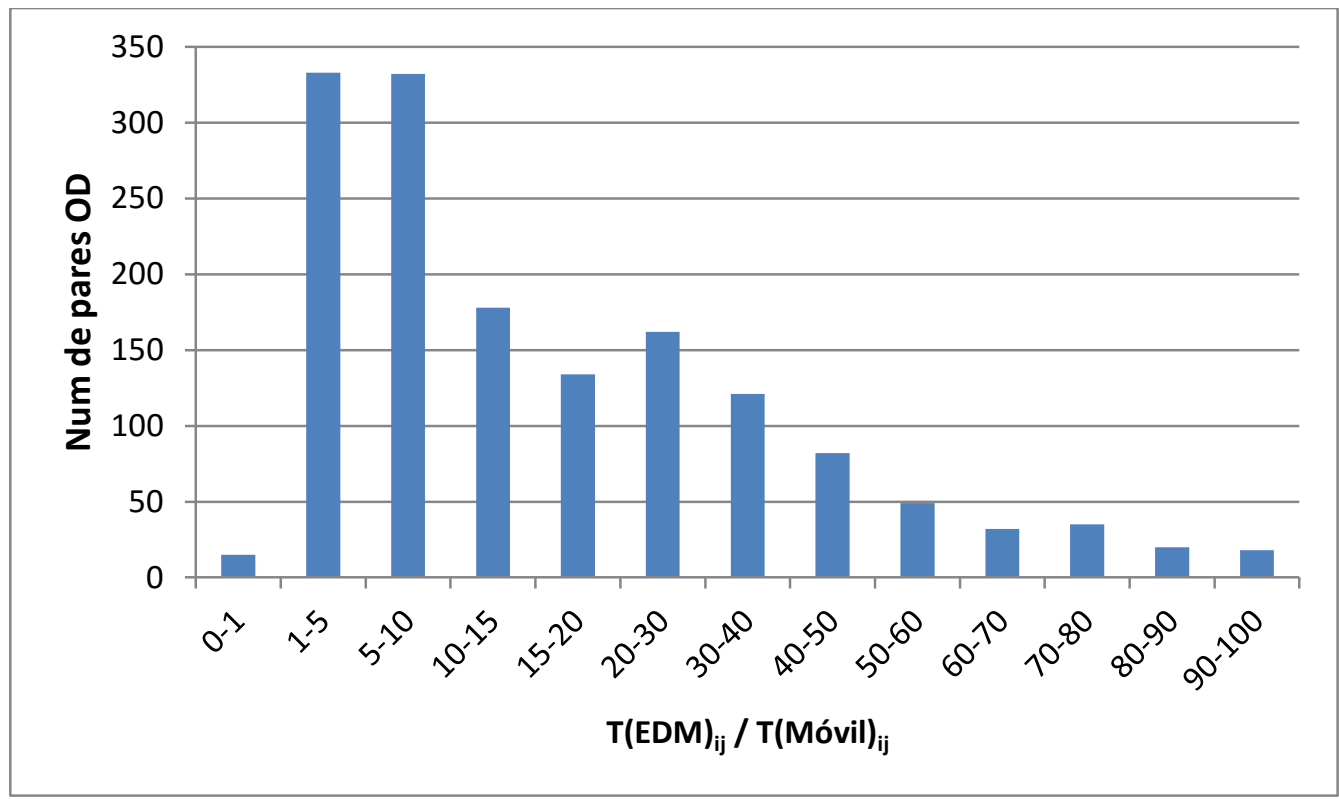

Fig. 1: Histograma de ratios entre los valores de las matrices de la EDM homogeneizada y del operador móvil para pares Origen-Destino con valores en ambas matrices

Dicho efecto resulta evidente en la Figura 1, donde se comparan el flujo de vehículos provenientes de la matriz de la EDM homogeneizada y la inferida por el operador de telefonía en las parejas O-D donde ambas matrices presentan valores (en la región de estudio piloto analizada). Hay que destacar que el ratio de viajes totales de ambas matrices es de 1,92 considerando todos los viajes, y de 1,53 si se incluyen tan sólo los viajes de más de 5 $\mathrm{km}$, mientras que el ratio medio de los pares individuales es de 25,59. De estas cifras se deduce que la matriz de la encuesta concentra el número total de viajes en sus 1.954 pares Origen-Destino con un valor de viajes mayor que cero, con lo que dichos valores están sobreelevados al compararlos con los valores de las casillas de la matriz del operador móvil, que puede distribuir su total de viajes entre 17.606 casillas con valor distinto de cero.

\subsubsection{Matriz del operador}

Enfocando la atención en la matriz O-D de viajes correspondiente a la franja de hora punta de día laborable (8:00-8:59), derivada de la explotación de los datos del operador de telefonía 
y su posterior transformación a matriz equivalente de vehículos mediante el factor de ocupación 1,534 personas/veh (), obtenido en base al estudio Exp. T-CS 6003/PATO del que también se derivó la EDM. En esta franja horaria el número de pares O-D con valores distintos de cero en la matriz es de 17606. Esta cifra supone que esta matriz de telefonía distribuye los viajes en nueve veces más pares O-D que la procedente de la EDM2010 homogeneizada. Las virtudes de esta matriz son varias: relativamente rápida de obtener (procesamiento en un periodo de tiempo de alrededor de 2 meses) y económicamente muy competitiva frente a una nueva encuestación; está basada en observaciones de una muestra amplia de la población y no tiene riesgo de sesgo ni de una baja tasa de respuesta. Además, se considera que su estructura es más realista que la procedente de la EDM2010, que adolece de sesgo por sobreelevación y que se encuentra concentrada en un pequeño conjunto de pares. Sin embargo, por su naturaleza, la matriz de telefonía presenta deficiencias de representación de los viajes de corta duración.

\section{ALGORITMO DE AJUSTE DE MATRICES MEDIANTE FUENTES HETEROGÉNEAS}

Es esta sección se presenta un esquema de algoritmo de ajuste colaborativo para diversas fuentes de datos heterogéneos, sucintamente descritas a continuación:

- Matriz previa $H_{i j}$ proveniente de la EDM2010 homogeneizada de la que se obtienen estimaciones fiables de magnitudes globales, como el número total de viajes $T$ y los viajes originados por las ZTs, $O_{i}$. Presenta escasa fiabilidad en cuanto a la distribución de dichos totales en los pares O-D.

- Matriz $L_{i j}$ obtenida por el operador de telefonía móvil con una estructura muy completa, con información observada de viajes en un elevado número de pares O-D, aunque con un nivel de viajes totales por debajo al inferido mediante otras fuentes de información previas existentes.

- Conteos de vehículos en el periodo de estudio en 212 arcos de la red.

- Tiempos de viaje en los arcos de la red promediados con 22 mediciones obtenidas del servicio web Directions Service de Google Maps.

Debido a las posibles inconsistencias anteriormente comentadas del conjunto de aforos y la consecuente incompatibilidad del sistema de ecuaciones ¡Error! No se encuentra el origen de la referencia., se opta por plantear un problema de optimización para minimizar la discrepancia de los volúmenes de vehículos modelados por la matriz O-D con los conteos de tráfico, a la vez que se conserva la información previa. Para medir la discrepancia con los volúmenes observados se propone la distancia euclídea entre los volúmenes observados y los modelados por el proceso de asignación:

$$
F_{2}(v, \bar{v})=\frac{1}{2}(v-\bar{v}) \cdot(v-\bar{v})
$$

donde 


$$
v=[\Pi] \cdot t
$$

Como se ha argumentado en el apartado 2.4, el uso de los tiempos de viaje en los arcos de la red para la asignación simplifica el proceso, pudiéndose asimilar a un algoritmo de proporciones constantes, correspondiendo en este caso a proporciones observadas en la realidad, siendo la matriz O-D la que tiene que adaptarse a ellas y no su reparto entre las diferentes rutas. Para usar una asignación más realista, que no penalice rutas próximas a la del coste mínimo pero algo superiores a ella y poder repartir el flujo en el caso de rutas con igual coste, se usa un algoritmo de asignación que alcance un equilibrio de usuario estocástico en lugar del determinista.

El control de la distorsión de la matriz previa se realiza mediante restricciones lineales en lugar de con el operador funcional $F_{1}$. Se puede formular de forma similar a (3) sin más que definir el lagrangiano elevando de nuevo las restricciones a la función objetivo multiplicadas por sus respectivos multiplicadores (Doblas \& Benitez, 2005).

Para conseguir que los viajes generados por las diferentes zonas de transporte en la matriz ajustada se correspondan a los de la matriz de la EDM2010, $O_{i}$, se definen las siguientes restricciones:

$$
\sum_{\forall j \in Z} T_{i j}=O_{i} \quad \forall i \in Z
$$

Las restricciones expresadas por el sistema de ecuaciones (10) son muy habituales en la definición de un problema de distribución, en este caso acotado simplemente por orígenes. Se definen solo restricciones concernientes al origen porque estos valores se derivan de una encuesta domiciliaria, de la que se puede inferir con garantías de fiabilidad magnitudes basadas en el hogar. Con el sumatorio de todas las ecuaciones del sistema (10) se obtiene la expresión (15):

$$
\sum_{\forall i \in Z} \sum_{\forall j \in Z} T_{i j}=T
$$

donde $T$ representa el número de viajes totales de la matriz EDM2010 homogeneizada. Al ser por tanto (11) una combinación lineal de las expresiones (10) no es necesario imponer dicha restricción, que sería redundante y ya se verifica con el sistema (10).

Para mantener la estructura de la matriz del operador, se añaden un conjunto de restricciones que se denominarán en adelante "restricciones de histograma". Para su definición se requiere clasificar los pares O-D de la matriz atendiendo al tiempo de viaje, calculado a través del proceso de asignación descrito en 2.4. Se genera un vector de tiempos de viaje de los pares, y éste se clasifica en un número discreto de intervalos (bins). Los tiempos de viaje inferiores a 15 minutos se dividen en solo 3 grupos, de 5 minutos cada uno, mientras que los tiempos superiores a 15 admiten una desagregación mayor, completando al final un número de bins 
o intervalos $N B$. Se define una matriz de incidencia $[\Gamma]$, de dimensiones $N P \times N B$, donde $N P$ es el número de pares O-D. Con el producto de la matriz de incidencia y la matriz O-D en su formato de pseudo-vector se obtiene directamente el número de viajes para cada bin. Se impone que esos valores sigan la distribución original de la matriz del operador, con lo que se añade el siguiente conjunto de restricciones:

$$
[\Gamma] \cdot t=b
$$

donde se ha definido el vector de coeficientes $\boldsymbol{b}$ como el producto del número total de viajes $T$ y la proporción de viajes observada en la matriz del operador para cada intervalo:

$$
b_{k}=T \frac{[\boldsymbol{\Gamma}]_{k} \cdot \boldsymbol{l}}{[\mathbf{I}] \cdot \boldsymbol{l}}
$$

donde [I] representa la matriz unidad y $\boldsymbol{l}$ es la matriz del operador en formato de pseudovector, y el subíndice $k$ identifica la fila k-ésima de la matriz.

Finalmente el problema de ajuste de la matriz que mejor explique los volúmenes observados conservando la estructura de la información recabada queda formulado como el siguiente problema de optimización cuadrática:

$$
\begin{array}{rlr}
\text { Minimizar } & \frac{1}{2}(\boldsymbol{v}-\overline{\boldsymbol{v}}) \cdot(\boldsymbol{v}-\overline{\boldsymbol{v}}) \\
\text { s.a. } & \\
\boldsymbol{v} \quad=[\Pi] \cdot \boldsymbol{t} & \\
\sum_{\forall j \in Z} T_{i j} & =O_{i} \\
{[\boldsymbol{\Gamma}] \cdot \boldsymbol{t}} & =\boldsymbol{b} &
\end{array}
$$

Para la resolución del problema (14) se propone partir con una matriz inicial $M_{i j}$, definida por la expresión (19):

$$
M_{i j}=\frac{O_{i}}{\sum_{j} L_{i j}} \beta_{b} L_{i j} \delta_{i j, b}
$$

La ecuación (15) define la solución de máxima entropía al problema de distribución acotado por orígenes y por las restricciones del histograma (12), con lo que usándose como punto de partida del algoritmo de resolución del problema (14) se tiene una solución factible y puede utilizarse cualquier método de optimización no lineal con restricciones lineales de punto interior, como el algoritmo de Frank \& Wolfe (Frank \& Wolfe, 1956). Para su obtención puede utilizarse una generalización del método tradicional de Furness, doblemente acotado aunque en este caso la segunda acotación no es por destinos sino por la pertenencia a un bin o intervalo del histograma de tiempo de viaje- número de viajes. 


\section{AGRADECIMIENTOS}

Este trabajo se ha desarrollado gracias a la financiación del proyecto FEDER de la Unión Europea con título "DIURMOVIL: Dinámica de una ciudad. Caracterización de la Movilidad Urbana mediante Datos de telefonía móvil y otras fuentes de información heterogénea" del Programa Operativo FEDER de Andalucía 2011-2015. Asimismo, se desea mostrar agradecimiento a la Agencia de Obra Pública de la Junta de Andalucía (Consejería de Fomento y Vivienda), al Consorcio de Transporte Metropolitano del Área de Málaga, y al operador de telefonía móvil Telefónica-Movistar. Por último, uno de los autores (N. Cáceres) también agradece al Ministerio de Economía y Competitividad el soporte proporcionado a través del Programa de Ayudas Torres-Quevedo (PTQ-13-06428).

\section{REFERENCIAS}

DIAL, R. (1971). A probabilistic multipath traffic assignment which obviates path enumeration. Transportation Research. Vol. 5, pp. 83-111.

AOPJA (2015). DIURMOVIL: Dinámica de una ciudad. Caracterización de la Movilidad Urbana mediante Datos de telefonía móvil y otras fuentes de información heterogénea. Agencia de Obra Pública. Junta de Andalucía. Proyecto G-GI3001IDIK. Programa Operativo FEDER, Unión Europea, 2011-2015.

DOBLAS, J., Y F.G. BENÍTEZ (2005). An approach to estimating and updating origindestination matrices based upon traffic counts preserving the prior structure of a survey matrix. Transportation Research 39B, 565-591

FRANK, M. Y WOLFE, P. (1956). An algorithm for quadratic programming. Naval Research Logistics Quarterly 3 (1-2): 95-110

SHEFFI, Y. (1985). Urban Transportation Networks: Equilibrium Analysis with Mathematical Programming Methods. Prentice-Hall, Englewood Cliffs, New Jersey

WARDROP, J. (1952). Some theoretical aspects of road traffic research. Proceedings of the Institute of Civil Engineers, Part 2, 325-378

YANG, H., T. SASAKI, Y. IIDA Y Y. ASAKURA (1992). Estimation of origin-destination matrices from link traffic counts on congested networks. Transportation Research 26B, 417 434 